\title{
TRABAJO SOCIAL Y EL PLAN DE ALTA DEL HOSPITAL 1
}

\author{
César M. Garcés Carranza²
}

\section{Resumen}

El Trabajo Social y el plan de alta del hospital generalmente implican trabajar en colaboración con un grupo interdisciplinario de profesionales que consiste de médicos, enfermeros, terapistas respiratorios, terapistas físicos, nutricionistas, así como también con otros sistemas de apoyo de la comunidad (asilos de ancianos/rehabilitación física o respiratorio, agencias de atención doméstica en el hogar, agencias de equipos médicos). A la unidad pulmonar los pacientes son transferidos mayormente de la Unidad de Cuidados Intensivos ( $\mathrm{UCl}$ ) o de otras unidades para continuar el tratamiento. En esta unidad el trabajador social prepara al paciente con el plan de alta una vez que su condición médica ha sido controlada y puede regresar a su casa o a un asilo/centro de rehabilitación. Algunos pacientes pueden necesitar de servicios de atención en la casa, de equipo médico (muletas, andadores, silla de ruedas, oxígeno, etc.). [Descriptores: Trabajo Social, plan de alta en hospital, decisiones éticas, dilemas éticos, colaboración interdisciplinaria].

\section{Summary}

Social work and discharge planning in the hospital setting generally implies working in collaboration with an interdisciplinary team of doctors, nurses, physical therapists,

${ }^{1}$ Este artículo emerge de la experiencias del autor en las diferentes unidades del hospital Bronx Lebanon (unidad de emergencia, Unidad de Cuidados Intensivos, Unidades de Medicina, Cirugía y Pulmonar), Bronx, New York.

${ }^{2}$ Trabajador social, Bronx Lebanon Hospital Center, Bronx, New York. 
nutricionists, utilization review case managers, as well as with other community support systems outside the hospital (nursing homes, rehab centers, home care agencies, medical supplies agencies, etc.). In these units of the hospital, the social worker prepares patients for their discharge once their medical condition has been stabilized and the patients can return to their home or to a health care facility if so recommended by the interdisciplinary team. Some patients might be in need of home care services or medical equipment (wheelchairs, walker, oxygen, etc.). [Keywords: social work, discharge planning in hospital, ethical decisions, ethical dilemmas, interdisciplinary collaboration].

\section{Introducción}

El Bronx Lebanon Hospital Center está ubicado en el sur del Bronx, que es el distrito más pobre de los Estados Unidos de Norte América. Casi el 50 por ciento de la población vive bajo el nivel de pobreza. Abundan enfermedades crónicas tales como el SIDA, asma, diabetes, problemas psiquiátricos, problemas psicosociales, como el abuso de drogas, alcohol, prostitución, desempleo, falta de viviendas, actividades pandilleras, maltrato infantil, maltrato de ancianos, violencia doméstica. Estos son algunos de los problemas comunes en este Distrito (American Fact Finder, 2007). Las características demográficas de los pacientes son; 53 por ciento latinos, 42 por ciento negros, 2 por ciento blancos, 0.05 por ciento asiáticos (Bronx Lebanon Hospital Center, 2007).

El plan de alta del hospital es una herramienta importante para la evaluación, y coordinación de atención médica a través de los centros de atención médica, incluyendo hospitales, asilos de ancianos/rehabilitación, atención doméstica en la casa o servicios de hospicio/paliativo (Center for Medicare Advocay, Inc., 2009). Lo que actualmente constituye el plan de alta del hospital está en debate y con frecuencia depende del lugar. EI término quiere decir unir al paciente y familiares con los recursos disponibles para darle continuación de servicios (Education Resources Information Center, 1999-2012). El plan de alta en Trabajo Social es una nueva faceta de una extensa práctica de coordinación de servicios. Este término implica unir al individuo y familiares con los recursos disponibles fuera del hospital para así darle continuación de servicios al paciente (Education Resources Information Center, 1999-2012). 
La admisión al hospital con frecuencia trae síntomas de ansiedad, miedo y preguntas que responder a los pacientes, familiares y amigos. Los médicos y enfermeros dan atención médica, pero es el trabajador social quien da apoyo emocional a los pacientes y familiares mientras dura lo que puede ser un evento emocional traumático. Esta intervención es importante para garantizar un resultado positivo. El trabajador social está capacitado para trabajar con individuos y familias para ayudarlos a superar la crisis causada por la hospitalización. La función del trabajador social es variada y compleja, y en el trabajador social desempeña una función importante en el bienestar de los pacientes durante la hospitalización y hace más fácil la transición del regreso al hogar, asilo, o centros de rehabilitación.

\section{Trabajo Social en el hospital}

El objetivo principal del Trabajo Social incluye en asistir a los pacientes y familiares con el plan de alta, dándoles consejería con respecto al impacto de la enfermedad y hospitalización. Entre sus funciones se encuentran:

1. Facilita la participación de la familia con el personal médico.

2. Mantiene informada a la familia acerca de progreso del paciente.

3. Utiliza el proceso del plan de alta para ayudar al paciente y a la familia para su mejor entendimiento sobre la conexión entre la enfermedad y la tensión; y sobre el efecto de esta en el sistema familiar en conjunto.

El trabajador o trabajadora social posee la capacitación para ayudar a pacientes y familiares a que se ayuden y superen la crisis; asiste y orienta en adquirir los instrumentos necesarios para que puedan superar situaciones difíciles tales como:

1. Enfermedades crónicas.

2. Alcoholismo y drogadicción.

3. Problemas emocionales causados por la enfermedad del paciente.

4. Problemas de relaciones familiares causadas por la enfermedad y hospitalización.

5. Abuso de ancianos y violencia doméstica. 
6. Problemas psicosociales.

7. Problemas de vivienda/desamparados

8. Falta de seguro médico

9. Pena/luto

El proceso del plan de alta debe de empezar al momento de la admisión del paciente al hospital. Esto tiene el potencial de reducir errores innecesarios y retrasos. Popoviv y Kozak (2000) demuestran que el plan de alta ha sido estudiado por investigadores de enfermería durante varios años, especialmente desde que se implementaron los esfuerzos para recortar el tiempo de duración de la hospitalización. En respuesta al recorte de días de hospitalización, los investigadores informaron y reconocieron que existen vacíos entre el hospital y las agencias de la comunidad a cargo de la continuación de atención médica, envío de información entre los proveedores de servicios, educación sobre el plan de alta a los pacientes y familiares.

Según mencionado por Foster, Murff, Peterson, Gandhi y Bates (2003), existen informes de pacientes describiendo la falta de su inclusión en el proceso de alta, familiares haciendo preguntas sobre su capacidad para atender al paciente en la casa, falta de recursos en la comunidad, tales como salud pública o agencias de atención que puedan ayudar a los pacientes en su casa. Foster, Clark, Renard, Dupuis y Chandoh (2004), señalaron que casi un 25 por ciento de los pacientes que fueron dados de alta del hospital sufrieron reacciones de eventos que fueron asociados con incapacidad física, y la mitad requirió de servicios de salud adicionales. Eventos adversos incluyeron errores en las órdenes de medicamentos, infección, confusión acerca de la educación sobre el proceso del plan de alta, la falta de seguimiento a los problemas por resolver.

Muchas veces, la falta de coordinación y adaptación a los problemas psicosociales trae como consecuencia el retraso en el proceso del plan de alta. La recuperación del paciente, así como también la demora de los servicios disponibles después que el paciente ha sido dado de alta, son medidas relevantes de un resultado positivo del proceso de alta del hospital, y el trabajador social trae consigo una perspectiva única a este proceso. El trabajador (a) social desempeña una función importante al proporcionar y desarrollar un plan de alta seguro y económico para el hospital. El plan de alta 
debe desarrollarse basado en los niveles adecuados para la continuidad de la atención médica. También está incluido el intercambio de información sobre la condición y necesidades psicosociales del paciente con otros proveedores profesionales de servicios médicos o de servicios auxiliares. El Trabajo Social está en la obligación de abogar por la reinvención del proceso del plan de alta. En el actual sistema de salud, los pacientes son dados de alta antes de tiempo. La determinación de quién recibirá servicios será la responsabilidad de los profesionales de Trabajo Social y otros proveedores de salud.

En la medida que los centros de salud buscan maneras para reducir los costos en los servicios, los trabajadores sociales deberían de abogar para que el plan de alta no sea incluido en los recortes de presupuesto. Y es que, a través del plan de alta y de la anticipación e identificación de problemas psicosociales y necesidades de los pacientes, existe la posibilidad de que el hospital ahorre dinero mediante la prevención, seguridad y satisfacción de los pacientes (Hager, 2010).

El plan de alta puede ser más imperativo cuando los temas de justicia social son correlacionados a las diferencias culturales y éticas. De acuerdo con Hage y Kenny (2009), la extracción de la diversidad social y cultural en la educación sobre la atención médica puede tener una influencia negativa en las personas sobre su opinión del mundo actual. Los temas socioculturales concernientes a la atención o prevención de problemas médicos pueden estar relacionados a cómo una comunidad en particular define sus necesidades de atención médica. Por ejemplo, si una persona de otra cultura rehúsa servicios médicos debido a miedos o creencias sin fundamentos, esto puede afectar en la calidad de atención para este paciente y puede poner en riesgo su salud (Hager, 2009). Chadiha, Proctor, Monroe, Darkwa y Dore (1995) encontraron que, en comparacion a los pacientes blancos, los pacientes afroamericanos, dados de alta del hospital, utilizaron menos servicios formales y tuvieron más problemas con el plan de alta debido a la falsa creencia de la disponibilidad de ayuda.

Como profesión, el Trabajo Social debe de abogar por mejorar la educación con respecto al plan de alta al aplicar un marco de trabajo de intervención, y transformar los asuntos que afectan de manera negativa al plan de alta en sus organizaciones. Los profesionales del Trabajo Social 
deben: 1) intervenir para aliviar los síntomas de los problemas psicosociales, al familiarizarse con estos temas; 2) reflexionar sobre estos problemas y preguntarse ¿por qué los pacientes son dados de alta del hospital sin una adecuada educación sobre el plan de alta?; 3) escuchar a aquellos pacientes que son afectados por el problema y hagan preguntas que puedan retar a la estructura social actual, y explorar las verdaderas causas de estos problemas (Hager, 2010). La profesión de Trabajo Social debería desarrollar o tomar una ruta diferente de acción con respecto a la atención médica y a los problemas psicosociales. La transformación de la acción se fija en la raíz del problema y no se detiene para aliviar los síntomas. Como profesionales del Trabajo Social deberíamos transformar nuestras comunidades y asistir a los pacientes que son atendidos con planes de alta adecuados al darles el poder de abogar por ellos mismos y a ser consumidores independientes del sistema hospitalario.

\section{Decisiones éticas en el plan de alta}

Kadushin y Egan (2001), formularon que las decisiones éticas del plan de alta representan un proceso de acción de los dilemas éticos de Trabajo Social. El proceso consiste en determinar si existe conflicto, ya sea que haya ocurrido daño, y desarrollar un plan de acción. Los trabajadores y trabajadoras sociales tienen la obligación de mantener la confianza de sus clientes, pero si el cliente amenaza con quitarse la vida o la de otras personas, el trabajador social está en la obligación de reportarlo a las autoridades. Esto representa un conflicto entre la obligación de mantener la confidencialidad y principios del ser humano, y el de prevenir daño a otra persona. Reamer (2006) plantea que tomar decisiones éticas es un proceso, y los trabajadores (as) sociales deben considerar los valores, principios y niveles en este Código. Parece que cuando se presentan dilemas éticos, el nivel del Código con respecto a la colaboración interdisciplinaria y protocolo sería un tema crítico de referencia y podría ayudar a los trabajadores sociales cuando se encuentren en situaciones difíciles (Portia, 2012).

\section{Ejemplo}

Cuando el médico tratante le informa al profesional de Trabajo Social que un paciente será dado de alta, y necesitará de servicios de ayuda en la casa, la responsabilidad del trabajador (a) social es coordinar que los servicios estén disponibles para cuando el paciente salga y regrese a su 
casa. Si los servicios no están disponibles para ese día, el paciente no puede salir. Esta situación de retraso coloca al paciente en un nivel alterno de atención (quiere decir que el paciente no necesita de más atención médica, y por lo tanto, el hospital recibirá substancialmente menos dinero de pago del seguro) hasta que los servicios necesarios se coordinen. En este sentido, al médico tratante se le hace responsable, pero el trabajador social también comparte esa responsabilidad. $Y$ en el proceso, se atrae la atención de la administración.

\section{Destrezas necesarias del trabajador o trabajadora social para el plan de alta}

La experiencia en el hospital nos permite identificar las siguientes destrezas:

1. Habilidad de trabajar en colaboración con otros miembros del personal interdisciplinario que está directamente trabajando con el paciente.

2. Tener destrezas analíticas y clínicas de evaluación.

3. Habilidad de comunicación con el personal médico, pacientes, familiares y con los servicios de la comunidad.

4. Habilidad de colaborar profesionalmente con el personal interdisciplinario.

5. Habilidad de establecer una relación terapéutica con pacientes y familiares.

6. Abogar por los derechos de los pacientes, especialmente cuando se ha identificado problemas que puedan comprometer el plan de alta y pongan al paciente en peligro.

\section{Ejemplo}

El médico tratante puede reportar que una paciente anciana y frágil, quién vive sola y no tiene familia inmediata, está dada de alta y tiene planeado mandarla a su casa sin servicios. Después de evaluar las condiciones psicosociales de la paciente, el trabajador o trabajadora social determina que la paciente no tiene la habilidad de dirigir a una persona para que la atienda en la casa, y recomienda que el plan de alta se retrase hasta que se evalúe más detalladamente al problema y quizás, se pueda desarrollar un plan de alta alternativo y más adecuado para proteger al paciente. En casos como este, el deber ético 
del trabajador social es el de informar al médico tratante que el plan de alta inicial puede poner al paciente en riesgo, y abogar por uno más adecuado, inclusive si esto significa retrasar el plan de alta. Es precisamente, en situaciones como esta, que los trabajadores sociales comprueban y demuestran su valor profesional al poner las necesidades de los pacientes por encima de otras consideraciones.

\section{Dilemas Éticos}

Kadushin y Egan (2001) definen los dilemas éticos como aquellos que ocurren cuando es necesario escoger entre situaciones éticas que parecen ser contradictorias. Los dilemas éticos presentan conflictos entre profesionales del Trabajo Social, personal médico, personal de agencias, pacientes, supervisores y la organización. Los profesionales del Trabajo Social operan bajo extensos principios éticos los cuales se basan en seis tipos de valores: a) servicio, b) justicia social, c) dignidad y valor de la persona; d) importancia de las relaciones humanas,; e) integridad; $y$ f) competencia (NASW, Code of Ethics, 1999). De acuerdo con Cole y Sparks (2006), antes de tratar de identificar, entender, y hacer comentarios acerca de dilemas éticos, los profesionales del Trabajo Social deben examinar primero sus propios valores. Entender las diferencias entre los distintos tipos de valores individuales es muy importante en la relación de los profesionales con los pacientes.

Para Beauchamp y Childres (1994), el propósito de la ética en el sistema de salud no es nuevo. Desde los tiempos de Sócrates, la medicina ética ha estado interesada en la obligación ética de los profesionales de cumplir con las necesidades del enfermo y el herido. El desarrollo tecnológico en la medicina, el rápido desarrollo del control del sistema de salud en la distribución de reembolsos en los costos, y la expansión de los sistemas de información han contribuido al frecuente aumento y complejidad de los dilemas éticos para todos los profesionales de salud, incluyendo a los trabajadores sociales. Varios factores han tenido impacto en el aumento de los dilemas éticos en la atención médica. Mientras que los avances científicos en medicina ofrecen oportunidades en alternativas de tratamiento y prolongación de la vida, también han creado dilemas éticos relacionados con temas sobre la calidad de vida, consentimiento, decisiones sobre el final de la vida incluyendo el retiro del equipo médico, acceso a servicios médicos, recursos y lugares de 
atención (Blumenfield \& Lowe, 1987; Cosson \& Manning, 1997; Reamer, 1998). Estos adelantos han creado preguntas para los trabajadores y trabajadoras sociales en lo que se refiere al proceso de hacer decisiones médicas (Blumenfield \& Lowe, 1987). En el ambiente hospitalario de Trabajo Social no solo es importante el aspecto clínico y ético -que es el producto de la práctica- si no la demanda de una respuesta razonable hacia un análisis ético al tomar decisiones.

Los avances en la tecnología médica y asuntos relacionados a la ética afectan diariamente la práctica del Trabajo Social. Tradicionalmente, su función estaba enfocada en desarrollar un variado plan poshospitalario que reúna las necesidades médicas y sociales de los pacientes. Estos temas con frecuencia se le presentan al profesional de Trabajo Social con la tarea de iniciar decisiones éticas y demostrar competencia ética que, se puede lograr utilizando un proceso razonable para una resolución ética (Reamer, 1998; Boland, 2006).

El hecho de que haya aumentado la cantidad de pacientes que permanece por menos tiempo en el hospital, el plan inadecuado de alta, la disposición de problemas y retrasos en el plan de alta, crean conflictos éticos cuando los profesionales del Trabajo Social tratan de balancear las necesidades de los pacientes con las necesidades de la organización, compañías de seguros médicos, personal médico, y familiares de los pacientes (Abramson, 1981; Cummings \& Cockerman, 1997; Boland, 2006).

\section{Conclusión}

El plan de alta debe iniciarse en el momento en que el paciente es admitido al hospital, con inclusión de la familia en el proceso, así como la identificación de obstáculos y objetivos de dicho plan. Esto ayudaría a que los profesionales del Trabajo Social se enfoquen en identificar los problemas psicosociales de los pacientes. El inicio del plan de alta al momento de la admisión al hospital también ayudaría a reducir costos al prevenir readmisiones innecesarias y mejorar la satisfacción de los pacientes. Los temas de ética en el sistema de salud continúan siendo un reto profesional para el Trabajo Social. El proceso y estrategias para las resoluciones éticas necesitan estudiarse y aclararse para entender las opciones que los trabajadores y trabajadoras sociales utilizan cuando identifican ese tipo de situación. 
Con frecuencia los trabajadores o trabajadoras sociales tienen muchos casos que atender y cumplir con las fechas establecidas por la organización para coordinar servicios necesarios para los pacientes. El Trabajo Social con respecto al plan de alta tiene una función de mucha demanda, y tensión física y emocional. Trabajan con frecuencia con casos complejos relacionados con pacientes que llegan al hospital con múltiples problemas psicosociales y requieren evaluación e intervención inmediata. No es raro que los profesionales del Trabajo Social traten con casos de personas desamparadas, sin vivienda, con problemas de desempleo, falta de dinero, falta de seguro médico, historial delictivo, abuso de drogas y alcohol, víctimas de violencia doméstica, problemas psiquiátricos. Cualquiera de estos problemas, juntos o separados, pueden impedir o retrasar el plan de alta del hospital. Simultáneamente situaciones que parecen mundanas como cuando un paciente necesita dinero para transporte público, recetas médicas, zapatos, ropa o un bastón, pueden ser causa para el retraso en el plan de alta del hospital. De ahí la importancia de completar la evaluación de las necesidades psicosociales del paciente al inicio de la admisión al hospital.

Para que los profesionales del Trabajo Social progresen, se debe continuar con la redefinición y reconceptualización de Trabajo Social. Esto es,definir la función de esta disciplina dentro del cambio del sistema hospitalario con su orientación financiera, preservando los valores, conocimientos, destrezas y ética profesional. 


\section{Referencias}

Abramson, Margaret. (1981). Ethical dilemmas for social workers in discharge planning. Social Work in Health Care, 6(4), 33-42

Abramson, Margaret. (1983). A Model for organizing an ethical analysis of the discharge planning process. Social Work in Health Care, 9(1), 45-52

America FactFinder. (2009). U.S Census Bureau. Population estimates for New York State by County. Recuperado de Factfinder2Census. gov/ faces/tableservices/ jsfpages/productview.xht

Beauchamp, Tom L., \& Childres, James F. (1994). Principles of biomedical ethics ( $4^{\text {th }}$ ed.). New York: Oxford University Press.

Blumenfield, Susan, \& Lowe, Jane. (1987). A Template for analyzing ethical dilemmas in discharge planning. Health and Social work, 12 (1) 47-56

Boland, Katheleen. (2006). Ethical Decision-Making among Hospital Social Workers. Journal of Social Work Values and Ethics, 3 (1).

Center for Medicare Advocacy, Inc. (2009). Medicare and Discharge Planning: Think Through Your Needs. Recuperado de www. medicareadvocacy.org

Cossom, James. (1992). What do we know about social work ethics? The Social Worker, 60 (3) 1650171.

Chadiha, L., E., Morroe-Howell, N., Darkawa, O., \& Dore, P. (1995). Post hospital home care for African-American and White elderly. The Gerontologist, 35(2), 233 - 239.

Cole, Portia, L. (2012). You want me to do what? Ethical practice interdisciplinary collaborations. Journal of Social Work Values and Ethics, 9 (1), 35.

Cummings, Susan, \& Cockerman, Clifford. (1997). Ethical dilemmas in discharge planning for patients with Alzheimer's disease. Health and Social Work, 22(2), 101-108.

Department of Health and Human Services. (1997, December). Medicare Hospital Discharge Planning (OEI-02-94-00320). Recuperado de http://hhs.gov/oei-02-94-00320.pdf.

Department of Health and Human Services. (2004, August). Condition of participation: Discharge Planning (42 C. F. R. 482. 43). Recuperado de htt://law.justia.com/us/cfr/title 42/423.0.21.3.199.12html.

Foster, Alan, Peterson, Josh. F., Gandhi, Tejal, \& Bates, David, W. (2003). The incidence and severity of adverse events affecting patients after discharge from the hospital. Ann Intern Med., 138(3), 161-167. 
Foster, Alan. J., Clark, H. D., Menard, Alex, Dupuis, N., Chernish, Robert, \& Chandok, Natasha, Kahn Asmet; Walraven, Carl, V.(2004). Adverse events among medical patients after discharge from the hospital. Canadian Medical Association Journal, 170(3), 345-349.

Hager, Julia Sara. (2010). Effects of a discharge planning Intervention on Perceived Readiness for Discharge. St Paul-Minnesota: St. Catherine University.

Kadushin, Goldie, \& Egan, Marcia. (2001). Ethical Dilemmas in Health Care: A Social Work Perspective. Health and Social Work. 26 (3), 136-149.

National Association of Social Workers. (1996). Code of Ethics. Washington, DC: Author.

Popoviv, Jennifer R., \& Kozak, Lola J. (2000). National hospital discharge survey: Annual summary, 1998. Vital and health statistics, No.148. Hyattsville, MD: National Center for Health Statistics.

Reamer, Frederick. (1985). The emergence of bioethics in social work. Health and Social Work, 10(4), 271-281.

Spehar, Analisa. M., Campbell, Robert, R. Cherrie, C., Palacios, Polly; Scott, Donna, Baker, Jacquelyn, Brad B. Jornstand, \& Jay Wolfson. (2001). Seamless care: Safe patient's transitions from hospitals to home. Advances in Patient Safety, 1, 79-97.

Sparks, Joanne. (2006). Ethics and Social Work in Health Care. Inc. En S. Gehler \& T. A. Browne (Eds). Handbook of Health Social Work (pp. 43-69) Hoboken, NJ: Wiley and Sons. 